\title{
Digital controller design based on APF's vector resonance control
}

\author{
Gui Hongming $^{1, a}$, Du Xiaoran ${ }^{1, a}$, Xi Ziqiang ${ }^{2, a}$
}

School of electrical and electronic engineering, Hubei University of Technology

651909945@qq.com

Keywords: active power filter, vector resonant controller, digital design

\begin{abstract}
There are many factors that affect the performance of active power filter, which is a key factor for command current tracking control. Aiming at the deficiency of traditional PR controller, this paper puts forward an improved PR controller-vector resonant controller. The controller has the advantages of stronger anti-interference ability and greater gain at the resonance point, and it can improve the accuracy of harmonic compensation in the current compensation control. In this paper, the digital controller in the digital control system has been given a detailed design. Finally, the experimental results show that the control strategy can further reduce the harmonic content and improve the compensation accuracy.
\end{abstract}

\section{Introduction}

Current command tracking control is a key link of active power filter compensation current control. For the current tracking control algorithm has many different scholars study. Different control algorithms in practical applications have their own characteristics. And there are also some shortcomings. The following several common control algorithms are introduced. PI control algorithm is one of the most common control algorithms in engineering applications, but the active power filter device tracking the command current is often harmonic components, can not be achieved steady state. If the PR resonant controller is used to control current, the harmonic current control requires separate adjustment of $6 \mathrm{k}-1$ and $6 \mathrm{k}+1$ harmonic, which will increase controller design complexity and calculation amount. And the PR controller gain is often limited at the resonant point, which will inevitably influence of harmonic compensation precision. To improve the design of PR controller, the PR controller can be transformed into the form of the fundamental synchronous rotating coordinate reference system, that is, the form of the vector resonance controller. It's noticeable that $6 \mathrm{~N}+1$ of the second harmonic signal is corresponding to the harmonic component of the positive sequence and negative sequence. The harmonics current are reflected in $6 \mathrm{~N}$ harmonics compensation after the introduction of fundamental synchronous rotating coordinate transformation matrix.

In this paper, the active power filter using vector resonant controller current tracking control strategy is given. And vector resonant controller using the root locus method to select the control parameters in the digital control system of discrete is focused on. Finally, the experimental results of the prototype show that the active power filter operating principle and the use of the accuracy of the current control can be very good harmonic compensation, and effectively improve the accuracy of harmonic compensation.

\section{The Current control strategy of APF system under the vector resonant control}

It's need to use different forms of current control to control the fundamental current and harmonic current in the fundamental synchronous rotating coordinate system. An proportion control link can be increased in the vector resonant controller, which can be written in the following form:

$$
G_{P I-V P I}=K_{p 1}+\frac{K_{i 1}}{s}+\sum_{h=6 \cdots 30} 2 \frac{K_{p h} s^{2}+K_{r h} s}{s^{2}+\left(n \omega_{s}\right)^{2}}
$$

The parameters of each harmonic in the vector resonant controller VPI are expressed by Kph and Krh. Ki1 and Kp1 are the PI controller parameters which regulate the fundamental current.

The system control structure of the current control using the PI-VPI controller is shown in Figure 
1. The outer loop of the APF control system is a voltage loop, and the PI controller is used. The role of the voltage outer loop is mainly to control the DC side voltage of APF. The inner loop of the control system is the current loop, and the VPI - PI controller is adopted. The main effect of the current inner loop is that when the Umod and Us are acting on the output inductor, the current controller controls the tracking command current, and acts on the APF inverter to make the output PWM wave duty cycle change.



Fig. 1 the system control structure under the control strategy of PI-VPI

\section{Discrete design of VPI controller}

In the digital control system, VPI controller design method has a variety of different options, the key to find a suitable method to calculate the parameters of the controller. The parameters which are obtained by different methods, in turn, determine the ability of the controller to track the current. If the use of the general idea, first calculating the control parameters of the mathematical model of the controller and the continuous system is transformed to discrete system, which is feasible. But vector resonant controller has characteristics of specific compensating amplitude frequency gain which is very large. It will have an impact on performance for the resonant frequency of the slight offset. So the selection method of discretization is very important.

To overcome the above disadvantages, this paper according to the VPI controller in continuous domain design after discretization model can choose in the discrete domain to model pole zero and gain direct design, which can get the parameters of current controller in discrete domain directly. For the discrete control system, the VPI controller is the two order discrete controller. And the general form can be expressed as:

$$
G_{V P I}(z)=\frac{K(z-a)(z-b)}{z^{2}-2 \cos (n \omega T) z+1}
$$

$\mathrm{K}$ is the controller gain, $\mathrm{a}$ and $\mathrm{b}$ are the zeros of the controller, and the number of harmonics is expressed by n.

In order to show the control effect of the two order discrete controller (2), the following derivation is given. The mathematical model of the APF object with the numerator and denominator expressed in the form of $\mathrm{G}(\mathrm{z})=\mathrm{N}(\mathrm{z}) / \mathrm{D}(\mathrm{z})$, can be obtained:

$$
H_{\varepsilon}(z)=\frac{1}{1+G_{V P I}(z) G(z)}
$$

The pulse transfer function of the control system is expressed by $H_{\varepsilon}(z)$ in the formula (3).

$$
H_{\varepsilon}(z)=\frac{\left[z^{2}-2 \cos (n \omega T) z+1\right] D(z)}{\left[z^{2}-2 \cos (n \omega T) z+1\right] D(z)+K(z-a)(z-b) N(z)}
$$

Given a frequency of the input, if the signal is sinusoidal, then it is expressed as follows: 


$$
R(z)=\frac{A \sin (n \omega T) z}{z^{2}-2 \cos (n \omega T) z+1}
$$
is $n \omega$

In the above formula (5), the amplitude of the input signal is expressed in A, and the frequency

The closed loop system can be expressed as the error signal under the excitation of the signal:

$$
\varepsilon(z)=H_{\varepsilon}(z) R(z)
$$

If we get (4) and (5) into (6):

$$
\varepsilon(z)=\frac{A \sin (n \omega T) z \cdot D(z)}{\left[z^{2}-2 \cos (n \omega T) z+1\right] D(z)+K(z-a)(z-b) N(z)}
$$

To make system stable, all of the poles of closed-loop transfer function are not beyond the unit circle, then type (7) using $\mathrm{Z}$ domain of the final value theorem are:

$$
\lim _{k \rightarrow \infty} E(k)=\lim _{z \rightarrow 1}\left[\left(1-z^{-1}\right) \varepsilon(z)\right]=0
$$

The meaning of the formula (8) is that as long as the function is set in the stable control condition, the controller (2) can achieve the control effect of the steady state error of the signal to a certain frequency.

In the APF electric loop control system, a direct discrete VPI controller model is introduced, which is added to the zero order holder and lag one shot control. And the control block diagram is shown in Figure 2.

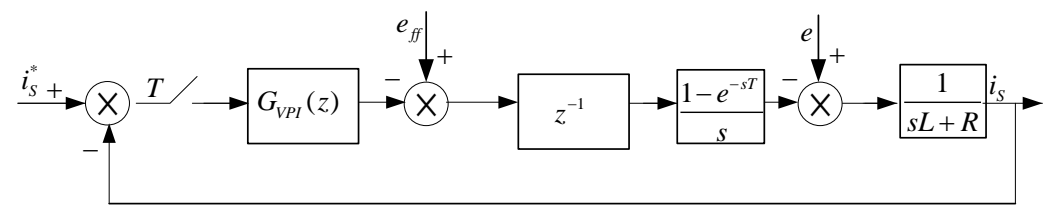

Fig. 2 VPI controller direct discrete circulation control system

According to figure 2, the current ring open loop transfer function:

$$
G_{0}(s)=\frac{K(z-a)(z-b)}{z^{2}-2 \cos (n \omega T) z+1} \frac{\left(1-e^{-(R / L) T}\right)}{z\left(z-e^{-(R / L) T}\right)} \frac{1}{R}
$$

Select $\mathrm{L}=0.25 \mathrm{mH}, \mathrm{R}=0.50, \mathrm{~T}=104 \mathrm{us}$, using the root locus method to give the specific design of the current controller. The 6 harmonic parameters as an example, if we put the parameter into the type (9), we can be obtained for open-loop transfer function as follow:

$$
G_{0}(s)=\frac{K(z-a)(z-b)}{z^{2}-0.616 z+1} \frac{0.3755}{z(z-0.8122)}
$$

In the open-loop transfer function (10), the Controller need to select the appropriate values of K, $\mathrm{a}, \mathrm{b}$. The value of $\mathrm{b}$ can firstly be set to 0.8122 , which will reduce the number of parameters set by the controller. The matlab is used to draw the root locus as shown in figure 3.Then we can get $\mathrm{a}=0.868, \mathrm{~K}=3.5227$.

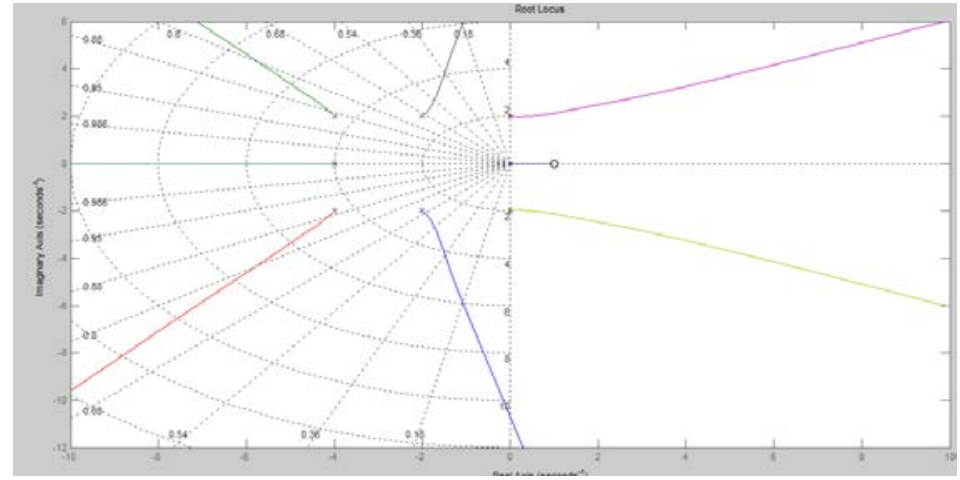

Fig. 3 root locus of zero and gain design 


$$
G_{V P I}(z)=\frac{3.5227(z-0.868)(z-0.8122)}{z^{2}-0.616 z+1}
$$

According to the various parameters can be obtained direct discretization design of VPI controller for each harmonic compensation.The current loop frequency response curves which compensate harmonic direct discretization of VPI controller are drawn as shown in Figure 4. From the figure the closed-loop response of the amplitude and the phase angle are 0 , indicating that the controller of each harmonic compensation has a good steady-state performance.



Fig. 4 current loop frequency response characteristics of the direct discrete VPI controller

\section{Experimental analysis}

The experiments study on a prototype of APF platform. The power measured value is about392V.The load is accessed through the resistance bridge series parallel (adjustable) composition through a rectifier bridge. The bus capacitor voltage is about $899 \mathrm{~V}$ monitoring in the debugging. The switching frequency of the pipe 9.6khz.The voltage space vector modulation (SVPWM) is used. The APF digital control system with PI-VPI controller is used to control the waveform.

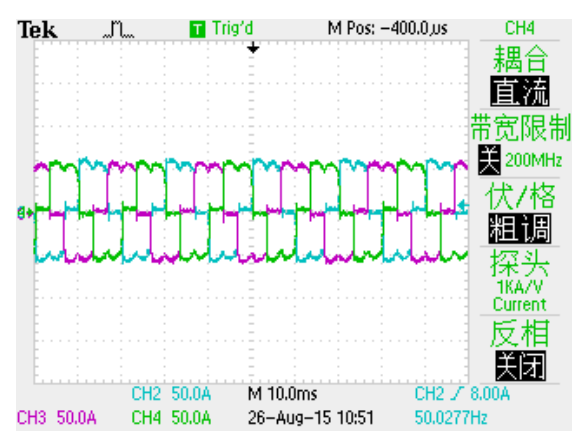

Fig. 5 current 53A of the load side 53A waveform

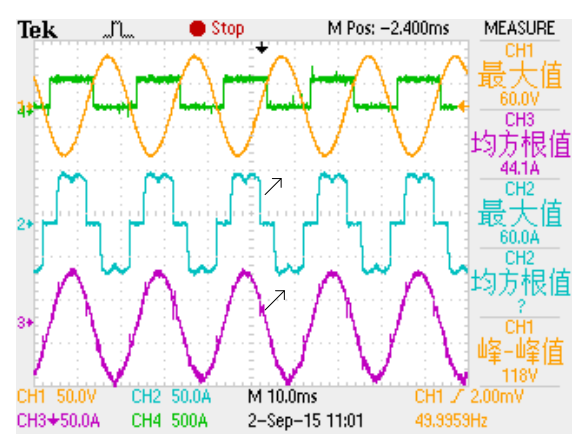

Figure 6 load current compensation waveform

Figure 5 and Figure 6 show the waveform of the load current before and after compensation. Where 1 represents the grid lateral line voltage waveform, 2 is on behalf of sampling phase-locked loop square waveform, 3 represents uncompensated current waveform, 4 is compensation to the current waveform. From the figure can be seen in this paper, the design of voltage phase locked loop (PLL) is very accurate, which can maintain and acquisition of current signal phase consistent. The current without compensation waveform seen from the load current distortion is very serious, which is approximate square wave waveform distortion. After the compensation ,the current waveform is a high degree of sine.Through the debugging can make the final THD reached 5\%. 


\section{Summary}

This paper mainly describes the fundamental synchronous rotating coordinate can control of harmonic component of the positive sequence and negative sequence harmonic components and get the vector resonant controller. The control structure in active power filter using vector resonant controller network side current control is given. Finally, from the introduction of the zero order holder in the digital control process, the influence of the sampling period on the controlled object is discussed. The parameters of the VPI controller are designed by using the root locus method. In the prototype of harmonic compensation experiment, the system current harmonic distortion rate of $5 \%$, harmonic compensation effect is good.

\section{Reference}

[1] D. Chen, J. Zhang, and Z. Qian, "An improved repetitive control scheme for grid-connected inverter with frequency-adaptive capability," IEEETrans. Ind. Electron., vol. 60, no. 2, pp. 814-823, Feb. 2013.

[2] M. Angulo. J. Lago. D. Ruiz-Caballcro. S. Mussa. and M. Hcldwein."Active power filter control strategy with implicit closed loop current control and resonant controller." IEEE Trans ,Ind. Electron., vol. 60. no. 7.pp. 2721-2730, Jul. 2013.

[3] Hamefors L, Yepes A G, Vidal A, et al. Passivity-based stabilization of resonant current controllers with consideration of time delay [J]. IEEE Transactions on Power Electronics, 2014, 29 (12): 6260-6263.

[4] Ricardo Lucio de Araujo Ribeiro, Thiago de Oliveira Alves Rocha,Raphaell Maciel de Sousa, Euzeli Cipriano dos Santos, Jr.. andAnlorjio_ Marcus jN0Queita_Li8ia. “A Robust DC-Link Voltage Control Strategy to Enhance the Performance of Shunt Active Power Filters Without Harmonic Detection Schemes" IEEE TRANSACTIONS ON INDUSTRIAL ELECTRONICS. VOL. 62. NO 2. Feb. 2015.

[5] Jinwei He, Yun Wei Li, Frede Blaabjerg, Xiongfei Wang,“Active Harmonic Filtering Using Current-Controlled, Grid-Connected DG Units With Closed-Loop Power Control, ”IEEE Trans. Power Electron, vol. 29, no. 2, pp.642-65,. Feb. 2014.

[6] M. Angulo. J. Lago, D. Ruiz-Caballero. S. Mussa, and M. Heldwein."Active power filter control strategy with implicit closed loop currentcontrol and resonant controller," IEEE Trans. Ind. Electron., vol. 6, no. 7,pp. 2721-2730, Jul. 2013.

[7] H. Yi, F. Zhuo, Y. Zhang. Y. Li, W. Chen, and J. Liu. "A source-current detccted shunt active power filter control scheme based on vector resonant controller," IEEE Trans. Ind. AppL, vol. 50, no. 3, pp. 1953-1965,Mav-Jun. 2014. 